\title{
hereditary hemochromatosis
}

$4 \quad$ Andrew M. Glazer, ${ }^{1}$ Lisa Bastarache, ${ }^{2}$ Lynn Hall, ${ }^{1}$ Laura Short, ${ }^{1}$ Tiffany Shields, ${ }^{1}$ Brett

$7 \quad{ }^{1}$ Department of Medicine, Division of Clinical Pharmacology, Vanderbilt University Medical Center, Nashville, TN, USA

$9{ }^{2}$ Department of Biomedical Informatics, Vanderbilt University Medical Center,

$11{ }^{3}$ Department of Pharmacology, Vanderbilt University Medical Center, Nashville, TN,

$16 *$ Correspondence should be addressed to Dan M. Roden, MD, Vanderbilt University

17 Medical Center, 2215B Garland Ave, 1285 MRBIV, Nashville, TN 37232. Email 


\section{Abstract}

23 Hereditary hemochromatosis $(\mathrm{HH})$ is an autosomal recessive disorder of excess

24 iron absorption. The most common form, $\mathrm{HH} 1$, is caused by loss of function variants in

25 HFE. HFE encodes a cell surface protein that binds to the Transferrin Receptor (TfR1),

26 reducing TfR1's affinity for the transferrin/iron complex and thereby limiting cellular

27 iron uptake. Two common missense alleles for HH1 have been identified, HFE C282Y

28 and HFE H63D; H63D is considered to be a less penetrant allele. When we deployed

29 Phenotype Risk Scores (PheRS), a method that aggregates multiple symptoms together in

30 Electronic Health Records (EHRs), we identified HFE E168Q as a novel variant

31 associated with HH. E168Q is on the same haplotype as H63D, and in a crystal structure

32 HFE E168 lies at the interface of the HFE-TfR1 interaction and makes multiple salt

33 bridge connections with TfR1. In in vitro cell surface abundance experiments, the $H F E$

$34 \mathrm{E} 168 \mathrm{Q}+\mathrm{H} 63 \mathrm{D}$ double mutation surprisingly increased cell surface abundance of HFE by

35 10-fold compared to wildtype. In coimmunoprecipitation experiments, however, HFE

36 C282Y, E168Q, and E168Q+H63D completely abolished the interaction between HFE

37 and TfR1, while H63D alone only partially reduced binding. These findings provide

38 mechanistic insight to validate the PheRS result that HFE E168Q is an HH1-associated

39 allele and lead to the reclassification of E168Q from a variant of uncertain significance to

40 a pathogenic variant, according to ACMG guidelines. HFE E168Q results in loss of HFE

41 function by disrupting the HFE-TfR1 interaction. In addition, some disease

42 manifestations attributed to H63D may reflect the functional effects of E168Q. 


\section{Introduction}

46 Hereditary hemochromatosis $(\mathrm{HH})$ is a genetic disorder in which the body absorbs

47 excess iron. ${ }^{1}$ The excess iron can cause damage to the body's tissues, resulting in a

48 myriad of symptoms, sometimes culminating in liver failure, heart failure, or diabetes.

$49 \mathrm{HH}$ has been linked to variants in multiple genes, including autosomal recessively

50 inherited variants in HFE (Hemochromatosis Type 1, HH1 [MIM 235200]). Two classic

51 common risk alleles for $\mathrm{HH} 1$ in $H F E$ are $\mathrm{C} 282 \mathrm{Y}$ and H63D. ${ }^{2,3}$ These missense variants

52 are common, with minor allele frequencies of $0-6 \%$ and $2-15 \%$ respectively, depending

53 on ancestry. ${ }^{4} \mathrm{C} 282 \mathrm{Y}$ homozygotes are considered to have the highest risk for $\mathrm{HH}$, with

54 C282Y heterozygotes, H63D homozygotes, and C282Y/H63D compound heterozygotes

55 at lower risk.,

56 HFE is a 343 amino acid single-pass transmembrane protein with a large

57 extracellular domain. The extracellular domain of HFE binds the extracellular domain of

58 Transferrin Receptor 1 (TfR1), reducing the affinity of TfR1 for transferrin. When HFE

59 is unable to bind TfR1 sufficiently, TfR1 has increased affinity for transferrin, resulting

60 in excess iron uptake into cells and hemochromatosis. C282Y eliminates a disulfide bond

61 in the extracellular domain of HFE, causing misfolding of HFE and failure to traffic to

62 the cell surface. ${ }^{3} \mathrm{H} 63 \mathrm{D}$ is thought to cause $\mathrm{HH}$ by having a smaller effect on reducing the

63 affinity of TfR1 for transferrin. ${ }^{2}$ Several additional rare variants have been observed in

64 patients with $\mathrm{HH}$ in the literature, typically as compound heterozygotes with $\mathrm{C} 282 \mathrm{Y}$ or

65 H63D. ${ }^{7-9}$

66 A promising method to evaluate the risk of genetic variants is in environments

67 that have relatively unselected populations with available Electronic Health Record 
68 (EHR) data and genotypic data. Recently, we have developed Phenotype Risk Scores

69 (PheRS) as a method to analyze syndromic phenotypes that have a range of phenotypic

70 effects and to link novel variants. The first deployment of PheRS in a biobank population

71 identified 18 associations between SNPs and syndromic diseases. ${ }^{10}$ One of the

72 associations was between HFE E168Q (c.502G>C) and hemochromatosis risk score. Out

73 of the 40 heterozygous carriers of HFE E168Q, 8 had highly elevated PheRS's for HH,

74 and 4 had received a liver transplant. However, whether E168Q actually alters HFE

75 function remains unclear. Largely due to the lack of in vitro functional data about its

76 mechanism, E168Q is still classified as a variant of uncertain significance. ${ }^{10}$

77 Here we investigate the function of HFE E168Q using in vitro functional assays.

78 We find that E168Q is located on the same haplotype as H63D, and that E168Q+H63D

79 has increased abundance at the cell surface. E168Q lies at the interface of the HFE-TfR1

80 interaction and completely disrupts that interaction, establishing a mechanism for

81 E168Q's association with hemochromatosis.

82

\section{Material and Methods}

$84 \quad$ Haplotype analysis

8552,573 adult ( $>18$ years) individuals of European ancestry were included in the analysis.

86 These individuals were genotyped with the Multi-Ethnic Global Array (Illumina) or the

87 Infinium HumanExome BeadChip array (Illumina). Genotypes were determined for $H F E$

88 H63D (rs1799945) or HFE E168Q (rs146519482). Ancestry was determined from

89 STRUCTURE. ${ }^{11}$ 
$91 \quad$ Mutagenesis and Transfection

92 pCB6-HFE-EGFP was a gift from Pamela Bjorkman (Addgene plasmid \# 12104). This

93 plasmid was mutated with a Quikchange Lightning Multi-site kit (Agilent) to create

94 C282Y, H63D, E168Q, and E168Q+H63D mutant plasmids. Plasmids were transfected

95 into HeLa, HepG2, Chinese Hamster Ovary, or HEK293 cells using Fugene 6 (Promega)

96 following manufacturer's instructions and studied 48-72 hours post-transfection.

$98 \quad$ Cell surface abundance assays

99 To stain cells for confocal and flow cytometry experiments, HeLa, HepG2, HEK293, or

100 CHO cells were stained with an anti-HFE antibody while still alive - thus, having an

101 intact cell membrane - to quantify HFE at the cell surface. Briefly, cells transfected with

102 wildtype or mutant HFE-GFP (see above) were trypsinized, resuspended in complete

103 media, washed in PBS+1\%Bovine Serum Albumin, incubated with a polyclonal anti-

104 HFE antibody at 1:500 dilution (ThermoFisher PA5-37364), washed twice in PBS+BSA,

105 incubated with a Alexa Fluor 647-anti-rabbit secondary antibody at 1:500 (ThermoFisher

106 A-21245), and washed twice in PBS+BSA. For flow cytometry, cells were analyzed on a

107 BD LSR Fortessa instrument, using a $488 \mathrm{~nm}$ laser and 525/50 nm filter for GFP, and a

$108633 \mathrm{~nm}$ laser and 660/20 filter for Alexa Fluor 647. Single cells were identified from side

109 and forward scatter parameters, and GFP and Alexa Fluor 647 laser levels were set so

110 that untransfected cells had a median of 100 for each. Cells with a high level of GFP were

111 identified (cells with $\sim 100$-fold GFP levels relative to wildtype; $10^{\wedge} 1.8$ to $10^{\wedge} 2.2$-fold

112 higher). The median Alexa Fluor 647 level of highly-GFP+ cells was calculated and

113 averaged across at least 3 replicate samples. Statistical analyses were performed in R. 
114 Student's two tailed t-tests were used for comparisons between groups. For confocal

115 microscopy, cells were stained as above, fixed with $4 \%$ paraformaldehyde, washed with

116 PBS+Hoechst, and imaged on an Olympus FV-1000 confocal microscope using identical

117 settings for each mutation.

118

119 Co-Immunoprecipitation

120 HeLa cells were chosen for coimmunoprecipitation experiments because wildtype HFE

121 trafficked best to the cell surface in HeLa cells (Figure S1) and they had been previously

122 used for coimmunoprecipitation experiments. ${ }^{9}$ HeLa cells were cotransfected using

123 Fugene 6 with wildtype or mutant $H F E$-GFP plasmids (see above) and a Transferrin

124 Receptor 1 expression plasmid pcDNA3.2/DEST/hTfR-HA, a gift from Robin Shaw

125 (Addgene plasmid \#69610). A Pierce Classic Magnetic IP/Co-IP Kit (ThermoFisher

126 \#88804) was used to harvest and coimmunoprecipitate the cells, following

127 manufacturer's instructions. Cells were precipitated with a rabbit anti-GFP antibody

128 (AbCam \#ab290), then Western blots were performed using the anti-GFP antibody

129 (1:2500) or a mouse anti-TfR1 antibody (ThermoFisher \#13-6800, 1:500) or a secondary

130 anti-rabbit HRP (Promega \#W4011) and anti-mouse HRP (Promega W-4021) antibody,

131 each at $1: 10,000$.

$133 \quad$ Variant classification

$134 H F E$ E168Q was classified according to American College of Medical Genetics and

135 Genomics criteria, ${ }^{12}$ which integrates multiple variables into a benign/likely

136 benign/uncertain significance/likely pathogenic/pathogenic classification. The University 
137 of Maryland Genetic Variant Interpretation Tool was used to implement these criteria

138 (medschool.umaryland.edu/Genetic_Variant_Interpretation_Tool1.html). ${ }^{13}$

\section{Results}

141 HFE E168Q is a rare allele on the same haplotype as H63D

142 HFE E168Q was previously shown using PheRS to have an association with

143 hemochromatosis. ${ }^{10}$ When we examined HFE genotypes of E168Q heterozygotes, we

144 observed that E168Q cosegregated with the common H63D allele (88/88 E168Q

145 heterozygotes had at least 1 H63D allele; Table 1). In individuals with European

146 ancestry, E168Q had a minor allele frequency of 0.00084 and H63D had a minor allele

147 frequency of 0.149 . E168Q was exclusively present in individuals of European ancestry,

148 except for 1 individual whose ancestry was undetermined by STRUCTURE, and is likely

149 of mixed ancestry. 13 of the 88 E168Q heterozygotes (14.7\%) were homozygous for

$150 \mathrm{H} 63 \mathrm{D}$, similar to the minor allele frequency of H63D, indicating that these individuals

151 were likely E168Q+H63D / H63D compound heterozygotes. Together, these data

152 indicate that E168Q is a rare variant that arose on the H63D allele of HFE.

$154 \quad$ HFE E168Q+H63D has increased abundance at the cell surface

155 A common mechanism for loss of function of membrane proteins is a defect in

156 trafficking to the cell surface, often due to misfolding of the protein and subsequent

157 aggregation along the secretory pathway. ${ }^{14}$ Cell surface abundance can also be affected

158 by altered rates of internalization or degradation. We developed a dual flow cytometry

159 and confocal microscopy-based assay to assay the subcellular localization of a GFP- 
160 tagged HFE protein. Testing of four cell lines (HeLa, HepG2, Chinese Hamster Ovary,

161 and HEK293) revealed that wildtype HFE trafficking efficiency varied widely between

162 cell lines, trafficking best in HeLa cells, followed by HepG2 cells (Figure S1). To test

163 whether mutant HFE proteins were present in different abundances at the cell surface, we

164 examined the cellular localization of GFP-tagged HFE wildtype protein and HFE mutants

$165 \mathrm{H} 63 \mathrm{D}, \mathrm{E} 168 \mathrm{Q}, \mathrm{C} 282 \mathrm{Y}$, and the E168Q+H63D double mutant (hereafter referred to as

166 E168Q+H63D) (Figure 1). C282Y showed a dramatic trafficking defect (5\% of wildtype

167 level, $\mathrm{p}=3.2 \mathrm{e}-5$, two-tailed $\mathrm{T}$ test). E168Q had a mild but significant increase in surface

168 abundance (136\% of wildtype level, $\mathrm{p}=0.01)$. H63D surprisingly had a higher abundance

169 than wildtype (722\% of wildtype level, $\mathrm{p}=0.02)$. E168Q+H63D also had a large increase

170 in surface abundance ( $970 \%$ of wildtype level, $\mathrm{p}=0.02)$. Similar relative surface

171 abundance results were observed in the HepG2 liver cell line, albeit with lower overall

172 levels of surface trafficking (Figures 1, S1). Therefore, in contrast to C282Y, H63D and

173 H63D+E168Q have increased cell surface abundances.

175 HFE E168 is at the interface of the HFE-Transferrin Receptor interaction

176 We next examined the location of E168Q within the HFE protein. A crystal

177 structure of the HFE-TfR1 interaction has been solved, together with the HFE binding

178 partner B2M (Figure 2). ${ }^{15}$ That crystal structure revealed six residues of HFE that made

179 salt bridges with TfR1 (Table S1). Intriguingly, in the structure, one of these residues is

180 HFE E168, which is located at the HFE-TfR1 interface, and makes salt bridges with two

181 TfR1 residues (TfR1-R629 and TfR1-Q640). HFE E168Q is also predicted to make an 182 intramolecular salt bridge with HFE N108. 
184 HFE E168Q disrupts the interaction between HFE and the Transferrin Receptor

185 Because of HFE E168's location and contacts with TfR1, we hypothesized that

186 the HFE E168Q variant disrupts the binding between HFE and TfR1. To test this, we

187 performed coimmunoprecipitation experiments, precipitating HFE-GFP using an anti-

188 GFP antibody and measuring coimmunoprecipitation of TfR1 (Figure 3). Wildtype HFE-

189 GFP coimmunoprecipitated TfR1, but C282Y, E168Q, and E168Q+H63D showed no

190 coimmunoprecipitation of TfR1. Across multiple replicates, C282Y had lower overall

191 intensity of anti-GFP staining in both input and immunoprecipitated samples, consistent

192 with a previously observed accelerated degradation rate of this variant. ${ }^{3}$ H63D showed a

193 detectable but decreased coimmunoprecipitation of TfR1. Thus, E168Q and

194 E168Q+H63D had a more severe defect in binding TfR1 than H63D.

196 Pathogenicity reclassification of $H F E$ E168Q

197 We used American College of Medical Genetics and Genomics criteria to

198 determine the classification of $H F E$ E168Q using data available before this study and

199 after this study (Table 2). Despite the genetic association in a biobank population, $H F E$

200 E168Q before this study was still classified as a variant of uncertain significance based

201 on criterion PS4 (variant prevalence in affected individuals is significantly increased

202 compared with the prevalence in controls). ${ }^{10}$ Based on the updated in vitro functional data

203 in this paper (criteria PS3), HFE E168Q now has enough evidence to be classified as a

204 pathogenic variant. 


\section{Discussion}

207 Phenotype Risk Scores for syndromic traits

208 HFE E168Q was identified as a novel HH1 allele with PheRS, a recently

209 developed method that combines multiple phenotypes into a weighted score, to study the

210 HH risk of different HFE variants. The PheRS for hemochromatosis includes 22

211 symptoms, such as liver cirrhosis, hepatic cancer, cardiac dysrhythmias, and type 2

212 diabetes. The in vitro work presented here validating E168Q as a loss of function allele

213 validates the use of PheRS as a powerful way to assess the disease risk of variants. As the

214 number of individuals in EHR datasets linked to genotyping grows, this approach will

215 gain in power to detect genetic associations. Given the size of contemporary biobanks

216 linking DNA variation to human phenotypes, ${ }^{16}$ this approach will likely prove fruitful for

217 rare but not ultrarare variants such as E168Q (minor allele frequency of $0.08 \%$ in

218 Europeans) that are still present in many individuals in large biobanks. However, for

219 some variants like HFE E168Q, statistical association in biobanks is not enough to

220 classify variants as pathogenic or likely pathogenic, and further in vitro functional

221 validation is required. The combination of statistical association by PheRS and in vitro

222 loss of function phenotype is enough to reclassify HFE E168Q as pathogenic.

223

224 Mechanism of HFE E168Q

225 A main mechanism of transmembrane proteins having loss of function is

226 misfolding and subsequent failure to traffic to the cell membrane. However, in this work,

227 we surprisingly observed that HFE E168Q+H63D had increased abundance at the cell

228 surface. Much of this surface abundance difference was due to H63D, although E168Q 
229 alone had a mild but significant increase in surface abundance. We observed that HFE

230 E168 was at the interface of the HFE-TfR1 interaction and made multiple salt bridge

231 contacts with TfR1, suggesting that E168Q would disrupt the salt bridge contacts with

232 TfR1. Indeed, coimmunoprecipitation experiments showed a complete loss of binding of

233 E168Q and E168Q+H63D. Although it is difficult to predict the exact configuration of

234 the mutant glutamine in the crystal structure, the glutamine likely completely disrupts the

235 interaction between HFE-168 and TfR1-R629 and likely alters the contacts with TfR1-

236 Q640. Two main alpha helices make contact with TfR1, termed $\alpha 1$ and $\alpha 2 .{ }^{15}$ HFE E168

237 is located in $\alpha 2$. Previous work showed that mutation to alanine of two residues in $\alpha 1$,

238 V100 and W103 (called V78 and W81 in the original paper), also abrogated the binding

239 between HFE and TfR $1 .{ }^{17}$ Therefore, we propose a model in which HFE E168Q is unable

240 to bind TfR1 and TfR1 therefore has an increased affinity for transferrin, causing iron

241 overload and HH1.

242

243 Improved prediction of $\mathrm{HH}$ risk

244 Our results suggest a template for improved prediction of $\mathrm{HH}$ risk. Our results

245 further suggest that genotyping for H63D and C282Y alone might not be sufficient to

246 determine $\mathrm{HH} 1$ risk. H63D is considered to be a low/variable penetrance HH1 allele, ${ }^{5,6}$

247 and E168Q presence may underlie some of the HH1 risk previously attributed to H63D

248 alone and explain some of its variable penetrance. ${ }^{18}$ Other rare HFE variants in $\alpha 1$ and $\alpha 2$

249 or making salt bridge connections with TfR1 may also disrupt TfR1 binding and lead to

250 hemochromatosis. Integrated phenotyping methods like PheRS show promise to identify

251 risk variants and may also identify patients with underrecognized disease. However, 
252 further functional studies are often necessary to validate these variants; $9 / 18$ variants in

253 the initial PheRS paper were classified as variants of uncertain significance despite their

254 statistical association with disease..$^{10}$ In vitro functional studies such as the surface

255 abundance and coimmunoprecipitation studies in this paper can validate the genetic

256 results and result in reclassification of variants as benign or pathogenic. We anticipate

257 that these methods will be more broadly applied to other variants, genes, and diseases to

258 better predict disease risk. 


\section{Supplemental Data Description}

260

261 The Supplemental Data contains 1 figure and 1 table.

262

263 Figure S1. HFE traffics robustly to the cell membrane in HeLa cells

264 Table S1. Residues of HFE forming salt bridges with TfR1

265

266 
bioRxiv preprint doi: https://doi.org/10.1101/547471; this version posted February 12,2019 . The copyright holder for this preprint (which was not certified by peer review) is the author/funder. All rights reserved. No reuse allowed without permission.

\section{Declaration of Interests}

268 The authors declare no competing interests. 


\section{Acknowledgements}

271 This work was supported by grants R01-LM010685 from the National Library of

272 Medicine and P50-GM115305 from the National Institute for General Medical Sciences

273 and a grant from the Robert J. Kleberg, Jr. and Helen C. Kleberg Foundation. BioVU

274 received and continues to receive support through the National Center for Research

275 Resources (UL1-RR024975), which is now the National Center for Advancing

276 Translational Sciences (UL1-TR000445). A.M.G. was supported by F32 HL137385 and

277 T32 HG008341. B.M.K. was supported by K99 HL135442. 


\section{Figure Legends}

281 Figure 1: HFE E168Q+H63D has enhanced surface abundance

282 HeLa or HepG2 cells were transfected with HFE-GFP and stained while alive for HFE at 283 the cell surface (Alexa Fluor 647). A) Representative confocal microscopic images of

284 HeLa cells (blue=Hoechst nuclear stain, green=GFP, red=Alexa Fluor 647). B)

285 Representative flow cytometry plots of surface HFE staining in HeLa cells. Red box 286 indicates transfected cells (GFP+ 100-fold higher than untransfected cells). X and Y-axis

287 scales are in $\log _{10}$ units. C-D) Quantification of flow cytometry data of cell surface

288 staining in HeLa (C) or HepG2 (D) cells (mean + SEM). Values are normalized to the

289 mean wildtype level. ${ }^{*} \mathrm{p}<0.05,{ }^{*} \mathrm{p}<0.005$, two-tailed $\mathrm{T}$ test. $\left.\mathrm{C}\right) \mathrm{n}=5-6$ replicate

290 samples/mutation. D) $\mathrm{n}=3$ replicate samples/mutation.

\section{Figure 2: HFE E168 is located at the interface of the HFE-TfR1 interaction}

293 A) Schematic of HFE/TfR1/B2M interactions at the cell surface. Orange/yellow rectangle

294 indicates plasma membrane. B-D) Crystal structure of HFE-TfR1-B2M extracellular

295 domains. ${ }^{15} \mathrm{HFE}$ is colored in salmon, TfR1 in green, B2M in blue. Each protein is present

296 twice in the complex, but only one instance is colored, and the other instance is shown in

297 gray. B) Side view. C) Top view with zoom. Salt bridges between HFE E168 and HFE

298 N108, TfR1 R629, and TfR1 Q640 are shown with dotted lines.

$300 \quad$ Figure 3: HFE E168Q disrupts the interaction between HFE and the Transferrin

\section{Receptor}


302 Coimmunoprecipitation experiments. A) Input. B) HFE-GFP was immunoprecipitated

303 with an anti-GFP antibody. For A and B, Western blots using anti-GFP or anti-TfR1 are

304 shown. Similar results were observed across 3 replicate experiments.

305

306 
307 Table 1: E168Q is a rare variant on the same haplotype as H63D

308

309

\begin{tabular}{|c|c|c|c|}
\hline & \multicolumn{3}{|c|}{ H63D allele } \\
\hline E168Q allele & HH & HD & DD \\
\hline EE & 38,084 & 13,256 & 1,145 \\
\hline EQ & 0 & 75 & 13 \\
\hline QQ & 0 & 0 & 0 \\
\hline
\end{tabular}

310 Co-occurrence of HFE H63D and E168Q genotypes in 52,573 adult European-ancestry

311 individuals. The 168Q allele only appears in the presence of the 63D allele, indicating

312 that the $168 \mathrm{Q}$ allele is on the same haplotype as the 63D allele.

313

314

315

316 


\section{Figure 1: HFE E168Q+H63D has enhanced trafficking to the cell surface}
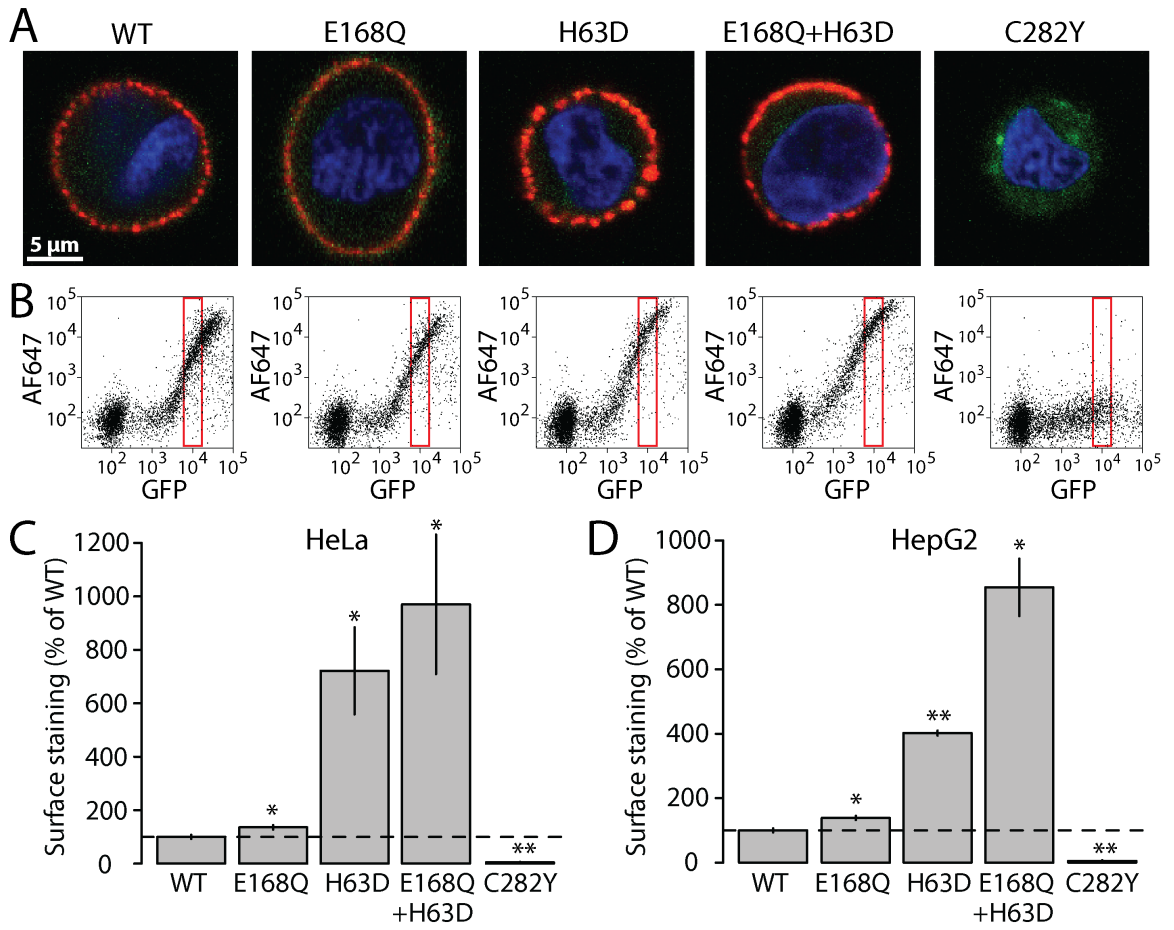

321 HeLa or HepG2 cells were transfected with HFE-GFP and stained while alive for HFE at

322 the cell surface (Alexa Fluor 647). A) Representative confocal microscopic images of

323 HeLa cells (blue=Hoechst nuclear stain, green=GFP, red=Alexa Fluor 647). B)

324 Representative flow cytometry plots of surface HFE staining in HeLa cells. Red box

325 indicates transfected cells (GFP+100-fold higher than untransfected cells). X and Y-axis

326 scales are in $\log _{10}$ units. C-D) Quantification of flow cytometry data of cell surface

327 staining in HeLa (C) or HepG2 (D) cells. Values are normalized to the mean wildtype

328 level. ${ }^{*} \mathrm{p}<0.05,{ }^{*} \mathrm{p}<0.005$, two-tailed T test. C) $\mathrm{n}=5-6$ replicate samples/mutation. D)

$329 \mathrm{n}=3$ replicate samples/mutation. 

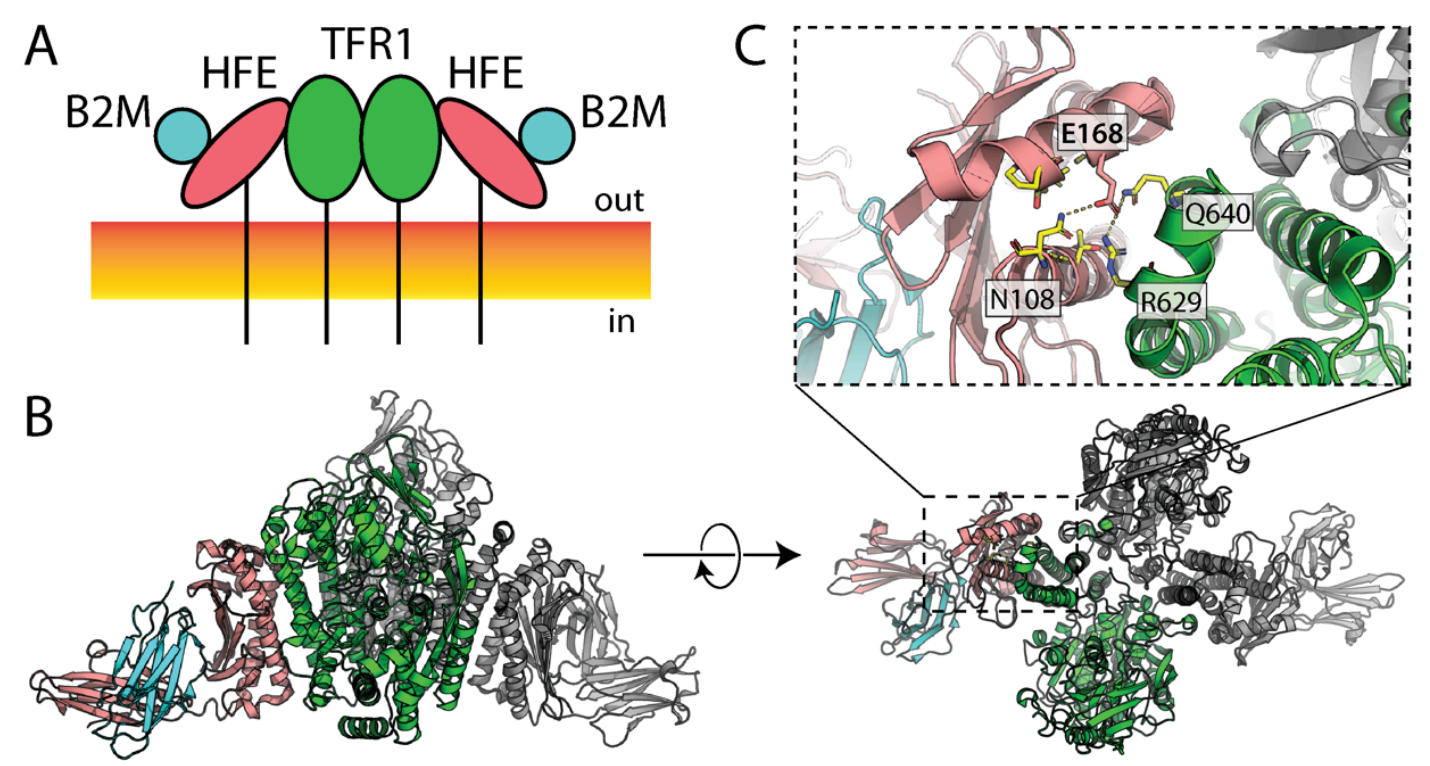

336 A) Schematic of HFE/TfR1/B2M interactions at the cell surface. Orange/yellow rectangle

337 indicates plasma membrane. B-D) Crystal structure of HFE-TfR1-B2M extracellular

338 domains ${ }^{15} \mathrm{HFE}$ is colored in salmon, TfR 1 in green, B2M in blue. Each protein is present

339 twice in the complex, but only one instance is colored, and the other instance is shown in

340 gray. B) Side view. C) Top view with zoom. Salt bridges between HFE E168 and HFE

341 N108, TfR1 R629, and TfR1 Q640 are shown with dotted lines. 


\section{Figure 3: HFE E168Q disrupts the interaction between HFE and the Transferrin}

\section{Receptor}

345

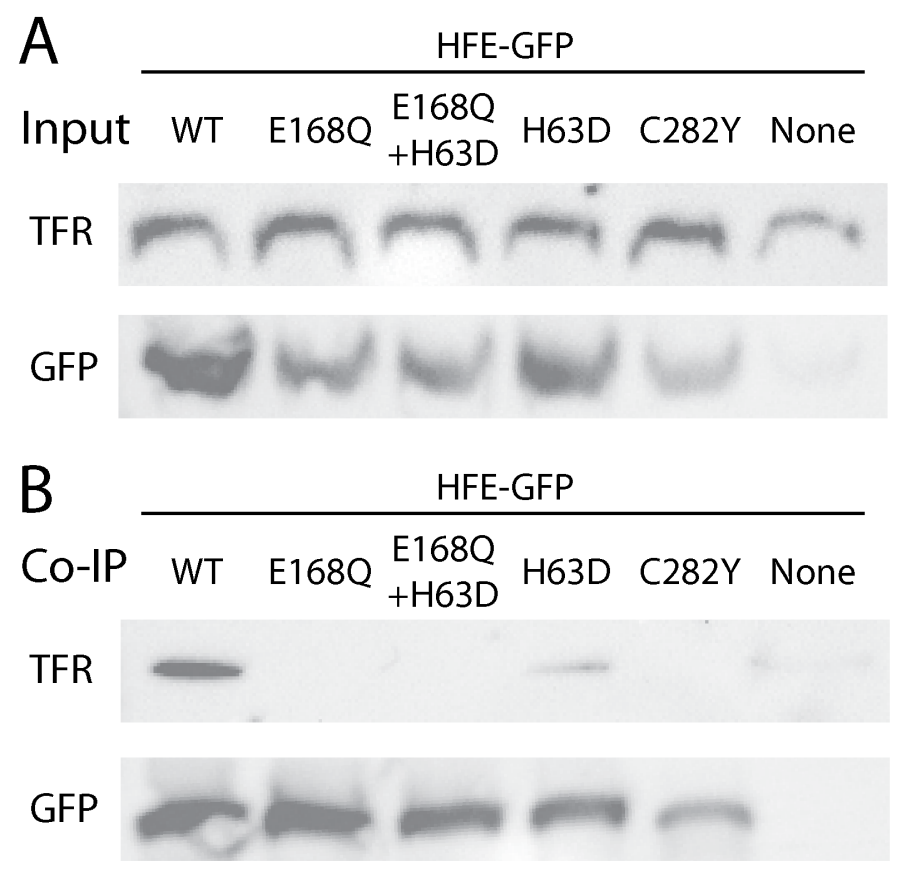

348 Coimmunoprecipitation experiments. A) Input. B) HFE-GFP was immunoprecipitated

349 with an anti-GFP antibody. For A and B, Western blots using anti-GFP or anti-TfR1 are

350 shown. Similar results were observed across 3 replicate experiments. 


\section{Table 2. Classification of HFE E168Q using ACMG guidelines}

354

\begin{tabular}{cccc}
\hline $\begin{array}{c}\text { ACMG } \\
\text { Criterion }\end{array}$ & Description & $\begin{array}{c}\text { Before } \\
\text { this study }\end{array}$ & $\begin{array}{c}\text { After this } \\
\text { study }\end{array}$ \\
\hline PS3 & $\begin{array}{c}\text { Well-established in vitro or in vivo functional } \\
\text { studies supportive of a damaging effect on } \\
\text { the gene or gene product }\end{array}$ & No & Yes \\
PS4 & $\begin{array}{c}\text { Variant prevalence in affected individuals is } \\
\text { significantly increased compared with the } \\
\text { prevalence in controls }\end{array}$ & Yes & Yes \\
\hline Classification & & VUS & Pathogenic \\
\hline
\end{tabular}

356 American College of Medical Genetics and Genomics (ACMG) classification

357 guidelines ${ }^{12}$ were implemented using the online Genetic Variant Interpretation Tool. ${ }^{13}$

358 Because of the in vitro functional evidence in this study, the classification of E168Q

359 changed from Variant of Uncertain Significance (VUS) to Pathogenic. 


\section{Figure S1. HFE traffics robustly to the cell membrane in HeLa cells}

362

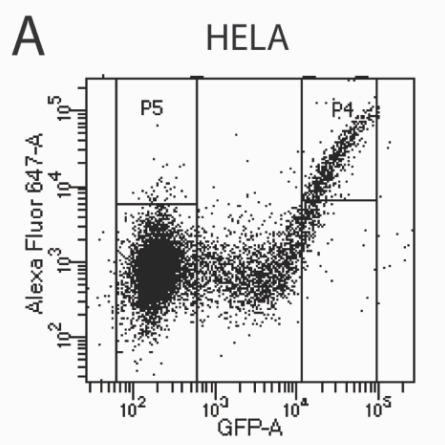

B HEK293
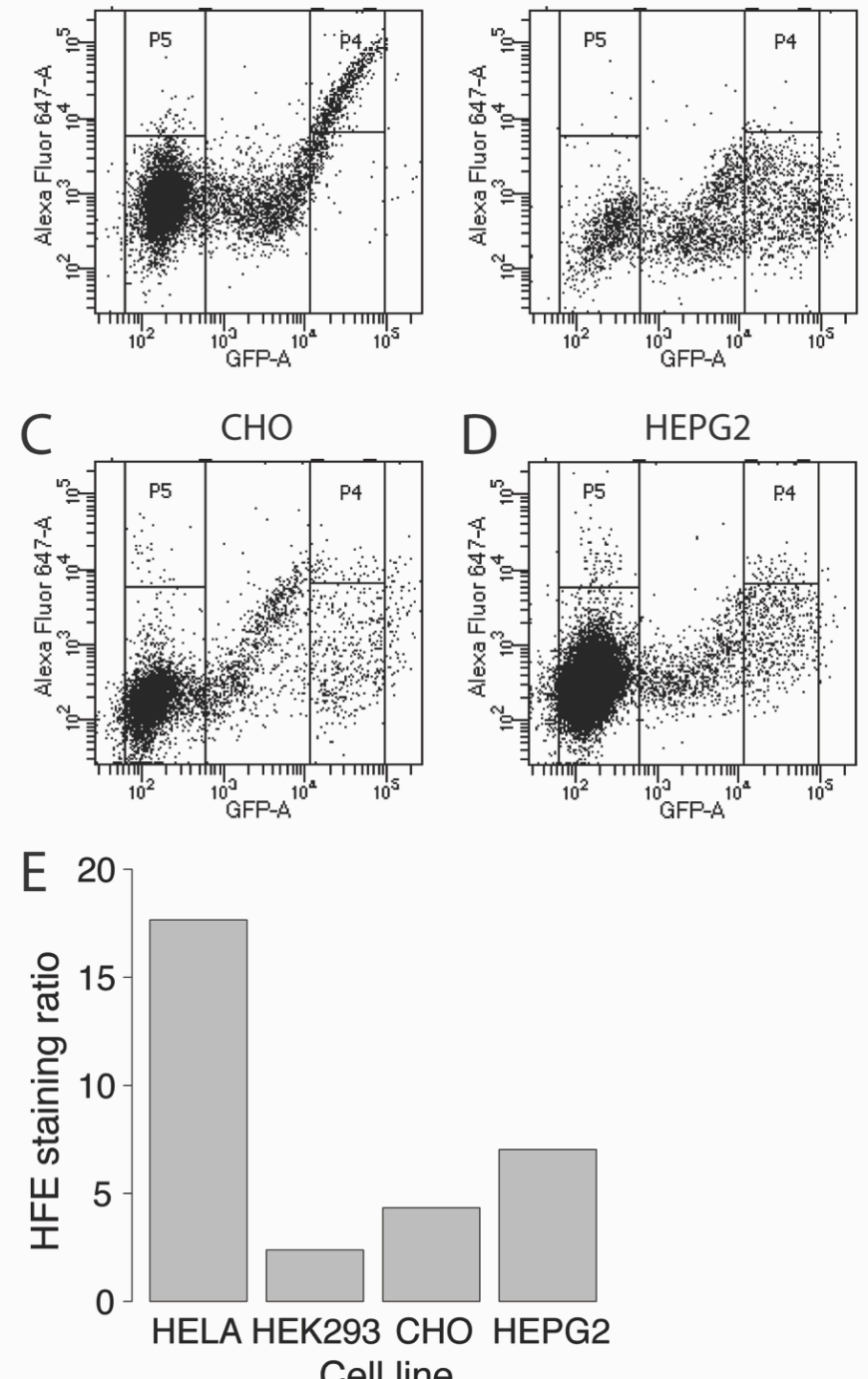

Cell line

366 A-D) Wildtype HFE cell surface staining (Alexa Fluor 647) vs. HFE-GFP level (GFP) in

367 transiently transfected HeLa (A), HEK293 (B), CHO (C), or HepG2 cells (D). E) A HFE

368 staining ratio was calculated by taking the median Alexa Fluor 647 level in highly

369 expressing cells (P4) divided by the median Alexa Fluor 647 level in untransfected cells 370 (P5). 
371 Table S1. Residues of HFE forming salt bridges with TfR1

372

\begin{tabular}{cccc}
\hline $\begin{array}{c}\text { HFE residue } \\
\text { (contemporary } \\
\text { numbering) }\end{array}$ & $\begin{array}{c}\text { HFE residue } \\
\text { (Bennett } \text { et al } \\
\text { numbering) }\end{array}$ & HFE location & $\begin{array}{c}\text { Interacting TfR1 } \\
\text { residue }\end{array}$ \\
\hline Gln86 & Gln64 & $\alpha 1$ helix & Thr658 \\
Glu107 & Glu85 & $\alpha 1$ helix & Arg629 \\
Asn108 & Asn86 & $\alpha 1$ helix & Arg629 \\
Glu168 & Glu146 & $\alpha 2$ helix & Arg629, Gln640 \\
Arg175 & Arg153 & $\alpha 2$ helix & Gln640 \\
Gln178 & Gln156 & $\alpha 2$ helix & Asp648 \\
\hline
\end{tabular}

373

374 Table adapted from Bennett et al. 2000. ${ }^{15}$

375 
1. Powell LW, Seckington RC, Deugnier Y. Haemochromatosis. Lancet. 2016;388:706-716. PMID: WOS:000381268700031.

2. Feder JN, Gnirke A, Thomas W, Tsuchihashi Z, Ruddy DA, Risch NJ, Bacon BR, Wolff RK. A novel MHC class I-like gene is mutated in patients with hemochromatosis. Blood. 1996;88:2579-2579. PMID: WOS:A1996VT98302579.

3. Waheed A, Parkkila S, Zhou XY, Tomatsu S, Tsuchihashi Z, Feder JN, Schatzman RC, Britton RS, Bacon BR, Sly WS. Hereditary hemochromatosis: Effects of C282Y and H63D mutations on association with beta(2)microglobulin, intracellular processing, and cell surface expression of the HFE protein in COS-7 cells. P Natl Acad Sci USA. 1997;94:12384-12389. PMID: WOS:A1997YF39300027.

4. Lek M, Karczewski KJ, Minikel EV, Samocha KE, Banks E, Fennell T, O'Donnell-Luria AH, Ware JS, Hill AJ, Cummings BB, Tukiainen T, Birnbaum DP, Kosmicki JA, Duncan LE, Estrada K, Zhao F, Zou J, Pierce-Hoffman E, Berghout J, Cooper DN, Deflaux N, DePristo M, Do R, Flannick J, Fromer M, Gauthier L, Goldstein J, Gupta N, Howrigan D, Kiezun A, Kurki MI, Moonshine AL, Natarajan P, Orozco L, Peloso GM, Poplin R, Rivas MA, Ruano-Rubio V, Rose SA, Ruderfer DM, Shakir K, Stenson PD, Stevens C, Thomas BP, Tiao G, Tusie-Luna MT, Weisburd B, Won HH, Yu D, Altshuler DM, Ardissino D, Boehnke M, Danesh J, Donnelly S, Elosua R, Florez JC, Gabriel SB, Getz G, Glatt SJ, Hultman CM, Kathiresan S, Laakso M, McCarroll S, McCarthy MI, McGovern D, McPherson R, Neale BM, Palotie A, Purcell SM, Saleheen D, Scharf JM, Sklar P, Sullivan PF, Tuomilehto J, Tsuang MT, Watkins HC, Wilson JG, Daly MJ, MacArthur DG, Exome Aggregation C. Analysis of protein-coding genetic variation in 60,706 humans. Nature. 2016;536:285-291. PMID: 27535533. PMCID: PMC5018207

5. Allen KJ, Gurrin LC, Constantine CC, Osborne NJ, Delatycki MB, Nicoll AJ, McLaren CE, Bahlo M, Nisselle AE, Vulpe CD, Anderson GJ, Southey MC, Giles GG, English DR, Hopper JL, Olynyk JK, Powell LW, Gertig DM. Iron-overloadrelated disease in HFE hereditary hemochromatosis. $N$ Engl J Med. 2008;358:221-230. PMID: 18199861.

6. Beutler E, Felitti VJ, Koziol JA, Ho NJ, Gelbart T. Penetrance of 845G--> A (C282Y) HFE hereditary haemochromatosis mutation in the USA. Lancet. 2002;359:211-218. PMID: 11812557.

7. Aguilar-Martinez P, Grandchamp B, Cunat S, Cadet E, Blanc F, Nourrit M, Lassoued K, Schved JF, Rochette J. Iron overload in HFE C282Y heterozygotes at first genetic testing: a strategy for identifying rare HFE variants. Haematologica. 2011;96:507-514. PMID: 21228038. PMCID: PMC3069226

8. Cezard C, Rabbind Singh A, Le Gac G, Gourlaouen I, Ferec C, Rochette J. Phenotypic expression of a novel C282Y/R226G compound heterozygous state in HFE hemochromatosis: molecular dynamics and biochemical studies. Blood Cells Mol Dis. 2014;52:27-34. PMID: 23953397. 
421 9. Silva B, Martins R, Proenca D, Fleming R, Faustino P. The functional

422

423

424

425

426

427

428

429

430

431

432

433

434

435

436

437

438

439

440

441

442

443

444

445

446

447

448

449

450

451

452

453

454

455

456

457

458

459 significance of E277K and V295A HFE mutations. Br J Haematol.

2012;158:399-408. PMID: 22624560.

10. Bastarache L, Hughey JJ, Hebbring S, Marlo J, Zhao WK, Ho WTT, Van Driest SL, McGregor TL, Mosley JD, Wells QS, Temple M, Ramirez AH, Carroll R, Osterman T, Edwards T, Ruderfer D, Edwards DRV, Hamid R, Cogan J, Glazer A, Wei WQ, Feng QP, Brilliant M, Zhao ZZJ, Cox NJ, Roden DM, Denny JC. Phenotype risk scores identify patients with unrecognized Mendelian disease patterns. Science. 2018;359:1233-+. PMID: WOS:000427504900035.

11. Pritchard JK, Stephens M, Donnelly P. Inference of population structure using multilocus genotype data. Genetics. 2000;155:945-959. PMID: WOS:000087475100039.

12. Richards S, Aziz N, Bale S, Bick D, Das S, Gastier-Foster J, Grody WW, Hegde M, Lyon E, Spector E, Voelkerding K, Rehm HL, Committee ALQA. Standards and guidelines for the interpretation of sequence variants: a joint consensus recommendation of the American College of Medical Genetics and Genomics and the Association for Molecular Pathology. Genet Med. 2015;17:405-424. PMID: 25741868. PMCID: PMC4544753

13. Kleinberger J, Maloney KA, Pollin TI, Jeng LJ. An openly available online tool for implementing the ACMG/AMP standards and guidelines for the interpretation of sequence variants. Genet Med. 2016;18:1165. PMID:

26986878. PMCID: PMC5026899

14. Schlebach JP, Sanders CR. The safety dance: biophysics of membrane protein folding and misfolding in a cellular context. $Q$ Rev Biophys. 2015;48:1-34.

PMID: 25420508. PMCID: PMC4339315

15. Bennett MJ, Lebron JA, Bjorkman PJ. Crystal structure of the hereditary haemochromatosis protein HFE complexed with transferrin receptor. Nature. 2000;403:46-53. PMID: 10638746.

16. Bycroft C, Freeman C, Petkova D, Band G, Elliott LT, Sharp K, Motyer A, Vukcevic D, Delaneau O, O'Connell J, Cortes A, Welsh S, Young A, Effingham M, McVean G, Leslie S, Allen N, Donnelly P, Marchini J. The UK Biobank resource with deep phenotyping and genomic data. Nature. 2018;562:203-209. PMID: 30305743.

17. Lebron JA, Bjorkman PJ. The transferrin receptor binding site on HFE, the class I MHC-related protein mutated in hereditary hemochromatosis. J Mol Biol. 1999;289:1109-1118. PMID: WOS:000081196100033.

18. Dickson SP, Wang K, Krantz I, Hakonarson H, Goldstein DB. Rare variants create synthetic genome-wide associations. PLoS Biol. 2010;8:e1000294. PMID: 20126254. PMCID: PMC2811148 